\title{
Chemokine Receptor CXCR7 Is a Functional Receptor for CXCL12 in Brain Endothelial Cells
}

\author{
Yang Liu ${ }^{1 *}$, Eleanor Carson-Walter ${ }^{1}$, Kevin A. Walter ${ }^{1,2 *}$
}

1 Department of Neurosurgery, University of Rochester School of Medicine and Dentistry, Rochester, New York, United States of America, 2 Wilmot Cancer Center, University of Rochester School of Medicine and Dentistry, Rochester, New York, United States of America

\begin{abstract}
The chemokine CXCL12 regulates multiple cell functions through its receptor, CXCR4. However, recent studies have shown that CXCL12 also binds a second receptor, CXCR7, to potentiate signal transduction and cell activity. In contrast to CXCL12/ CXCR4, few studies have focused on the role of CXCR7 in vascular biology and its role in human brain microvascular endothelial cells (HBMECs) remains unclear. In this report, we used complementary methods, including immunocytofluorescence, Western blot, and flow cytometry analyses, to demonstrate that CXCR7 was expressed on HBMECs. We then employed short hairpin RNA (shRNA) technology to knockdown CXCR7 in HBMECs. Knockdown of CXCR7 in HBMECs resulted in significantly reduced HBMEC proliferation, tube formation, and migration, as well as adhesion to matrigel and tumor cells. Blocking CXCR7 with a specific antibody or small molecule antagonist similarly disrupted HBMEC binding to matrigel or tumor cells. We found that tumor necrosis factor (TNF)- $\alpha$ induced CXCR7 in a time and dose-response manner and that this increase preceded an increase in vascular cell adhesion molecule-1 (VCAM-1). Knockdown of CXCR7 resulted in suppression of VCAM-1, suggesting that the reduced binding of CXCR7-knockdown HBMECs may result from suppression of VCAM-1. Collectively, CXCR7 acted as a functional receptor for CXCL12 in brain endothelial cells. Targeting CXCR7 in tumor vasculature may provide novel opportunities for improving brain tumor therapy.
\end{abstract}

Citation: Liu Y, Carson-Walter E, Walter KA (2014) Chemokine Receptor CXCR7 Is a Functional Receptor for CXCL12 in Brain Endothelial Cells. PLoS ONE 9(8): e103938. doi:10.1371/journal.pone.0103938

Editor: Rajesh Mohanraj, UAE University, Faculty of Medicine \& Health Sciences, United Arab Emirates

Received April 22, 2014; Accepted July 6, 2014; Published August 1, 2014

Copyright: (C) 2014 Liu et al. This is an open-access article distributed under the terms of the Creative Commons Attribution License, which permits unrestricted use, distribution, and reproduction in any medium, provided the original author and source are credited.

Data Availability: The authors confirm that all data underlying the findings are fully available without restriction. All relevant data are within the paper.

Funding: This study was supported by the University of Rochester Clinical and Translational Science Institute Pilot Studies Award to Y.L., the National Institutes of Health (NINDS K08 NS046461) to K.A.W., the American Brain Tumor Association to K.A.W., and the Ronald Bittner Brain Tumor Research Fund to K.A.W. The funders had no role in study design, data collection and analysis, decision to publish, or preparation of the manuscript.

Competing Interests: The authors have declared that no competing interests exist.

* Email: yang_liu@urmc.rochester.edu (YL); kevin_walter@urmc.rochester.edu (KAW)

\section{Introduction}

Chemokine CXCL12 (stromal cell derived factor $1 \alpha$ or SDF- $1 \alpha$ ) is a key player in angiogenesis and impacts such diverse processes as inflammation, wound healing, embryonic development, and the growth of malignant tumors. CXCL12 promotes tubulogenesis and migration of endothelial cells, including human umbilical endothelial cells (HUVECs) and human microvascular endothelial cells (HMVEGs) [1-3], largely through binding chemokine receptor 4 (CXCR4) [4-7].

Recently, CXCR7 was recognized to be a second receptor for CXCL12 [8,9]. In contrast to the extensive studies regarding CXCR4, fewer studies have investigated the role of CXCR7 in endothelial biology. CXCR7 is expressed by human endothelial cells, including HUVECs [9-12], HMVEGs [13], pulmonary microvascular endothelial cells [14], and endothelial cells within the central nervous system [15]. CXCR7 $-/-$ mice die in the first week after birth due to abnormal cardiovascular development, suggesting a critical role for CXCR7 in endothelial biology and cardiac development [16,17]. We originally identified CXCR7 (then called RDC1) as a selective marker of glioblastoma (GBM) derived microvascular endothelial cells and confirmed that it was induced in the tumor endothelium of both primary and metastatic malignant brain tumors [18,19]. CXCR7 expression increased with brain tumor grade [20,21] and elevated CXCR7 mRNA levels correlate with poor survival in glioma patients (Liu Y, unpublished data). Aberrant CXCR7 expression has subsequently been reported in the vasculature of a variety of cancer models [2026], indicating that CXCR7 may actively contribute to tumor angiogenesis and/or vasculogenesis. However, the function of CXCR7 in brain endothelium has remained unclear. In this study, we investigated the role of CXCL12/CXCR7 signaling in human brain microvascular endothelial cells (HBMECs). We found that GXCR7 regulates multiple HBMEC functions including proliferation, tube formation, migration, adhesion, and binding to GBM cells. We further demonstrated that GXCR7 in HBMECs can be up-regulated by tumor necrosis factor (TNF)- $\alpha$ and is an upstream gene of VCAM-1.

\section{Materials and Methods}

\section{Cells and Reagents}

Primary human brain microvascular endothelial cells (HBMECs) were from Cell Systems (Kirkland, WA) and maintained in complete medium with serum and CultureBoost (Cell Systems). All primary HBMEGs cultures were used between passage 4 and 9. GBM tumor cells U251MG cells and U373MG cells were gifts of Dr. John Laterra (Johns Hopkins School of Medicine) and originally purchased from the American Type Culture Collection (ATCG). U251MG cells were maintained as 
previously described [27]. U373MG cells were maintained in DMEM (Invitrogen) with 10\% FBS, 20mM HEPES (Invitrogen) and 1\% penicillin-streptomycin. Primary CXCR7 antibody (11G8) and mouse IgG1 isotype control for immunocytofluorescence and neutralization experiments were from R\&D Systems (catalogue numbers: MAB42273 and MAB002, respectively). Primary CXCR7 and VCAM-1 antibodies for immunoblot analysis were from GeneTex (catalogue numbers: GTX100027 and GTX110684, respectively). Human CXCR7 phycoerythrin antibody and isotype control for flow analysis were from $\mathrm{R} \& \mathrm{D}$ Systems (catalogue numbers: FAB42271P and IC003P, respectively). Recombinant human CXCL12/SDF- $1 \alpha$ and TNF- $\alpha$ were from Peprotech (Rocky Hill, NJ).

\section{Immunocytofluorescence}

HBMEGs were plated on culture slides (BD, catalogue number: 354118 ) at a density of $6 \times 10^{4} /$ well on day 1 . On the second day, cells were washed with PBS, followed by fixation with $3.2 \%$ paraformaldehyde (PFA) at room temperature for $15 \mathrm{~min}$. Cells were blocked with 10\% normal donkey serum for 1 hour and incubated with primary antibody (11G8, 1:200) for 1 hour. Cells were washed with PBS and incubated with donkey anti-mouse Alexa Fluor 594 (1:1000; Invitrogen, catalogue number: A21203) for 1 hour, washed and mounted with Prolong Gold antifade reagent with DAPI (Invitrogen). Images were acquired on an Olympus IX71 inverted microscope with DP controller (3.2) software.

\section{Construction of shRNA-CXCR7 HBMEC}

GIPZ lentiviral shRNAmir plasmids expressing short hairpin RNA (shRNA) targeted to CXCR7 and scrambled control sequences were purchased from Thermo Scientific (Waltham, MA). Recombinant lentiviruses were produced by co-transfecting human embryonic kidney 293T cells with the GIPZ lentiviral vectors and translenti viral GIPZ packaging system (Thermo Scientific) using X-tremeGENE HP DNA transfection reagent (Roche Diagnostics, Indianapolis, IN) according to manufacturer's protocol. HBMECs were infected with lentiviruses at 0.5 multiplicity of infection, followed by selection with puromycin $(0.5 \mathrm{ug} / \mathrm{ml})$. Stably expressing shRNA-scramble and shRNACXCR7 lines were designated scramble-HBMECs $(\mathrm{SG})$ and p148-HBMECs (p148), respectively. Lack of sequence homology between CXCR7, CXCR4, VCAM-1 and RefSeq genomes was confirmed using BLAST (NIH) to ensure that all shRNA-CXCR7 constructs were specifically targeted to GXCR7.

\section{RNA isolation and reverse transcription}

Total RNA was isolated using Qiagen RNeasy Mini kit (Qiagen, Qiagen Sciences, MD) according to manufacturer's protocol. cDNA was generated using iScript cDNA Synthesis kit (Bio-Rad, Hercules, CA) according to manufacturer's protocol.

\section{Quantitative real-time PCR ( $q P C R$ )}

qPCR was performed by using iQ5 real-time PCR detection system (Bio-Rad) as previously described [19]. CXCR7 and GAPDH primers were previously reported [19]. CXCR4 forward primer: CGTCGTGCTGACTATTCGCGA, reverse primer: GGAACACAACCACCCACAAGT. Vascular cell adhesion molecule-1 (VCAM-1) primers were described in a previous publication [28]. All primers were synthesized by Integrated DNA Technologies (IDT, Coralville, IA). Samples were analyzed in triplicates.

\section{Western blotting}

Total protein was extracted from shRNA-CXCR7 or scramble HBMECs using RIPA buffer (Thermo Scientific) and quantified using Bio-Rad DC protein assay kit (Bio-Rad). Thirty micrograms of total protein was loaded on a Novex 10\% Tris-Glycine gel (Life Technologies), electrophoresed and transferred to a PVDF membrane. Blots were incubated with CXCR7 or VCAM-1 primary antibody $(1: 250)$ at $4^{\circ} \mathrm{C}$ overnight, followed by goat antirabbit horseradish peroxidase secondary antibody (1:2000) for 1 hour at room temperature and visualized with Immobilon Western Chemiluminescent HRP Substrate (Millipore). To control protein loading, blots were probed with goat anti-actin $1: 1000$,
A.

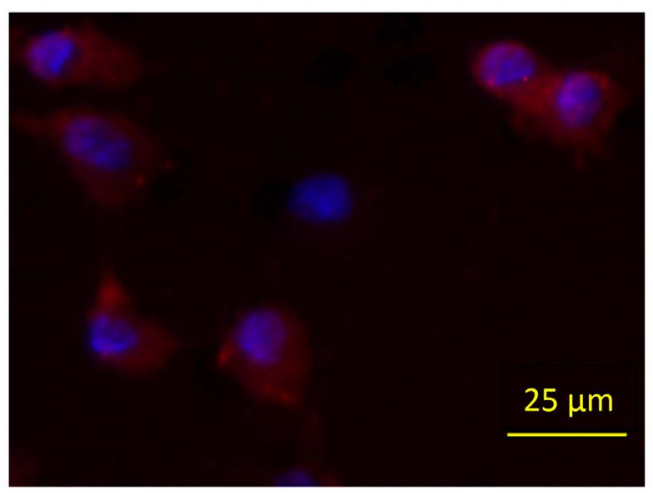

B.

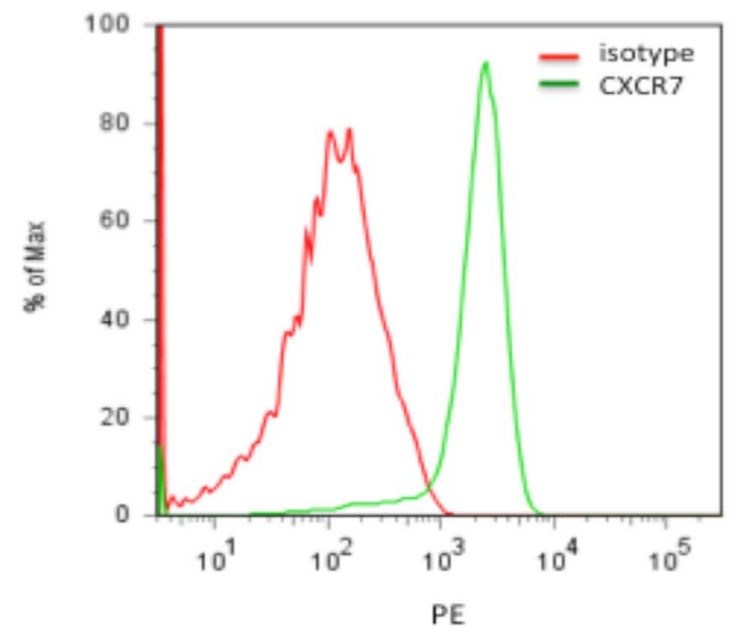

Figure 1. CXCR7 is expressed in HBMECs. A, Representative immunocytofluorescence image for CXCR7 (red) in HBMECs. Nuclei are stained with DAPI (blue). B, Flow cytometry analysis for CXCR7 in HBMECs. HBMECs were stained with CXCR7 specific primary antibody and subjected to immunocytofluorescence and flow cytometry, respectively, as described in "Materials and Methods". doi:10.1371/journal.pone.0103938.g001 
A.

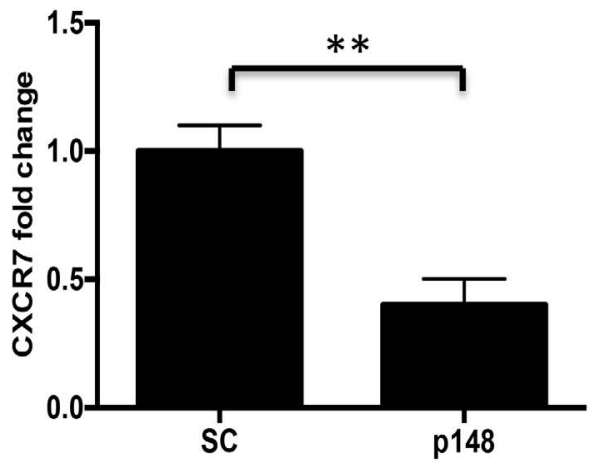

B.

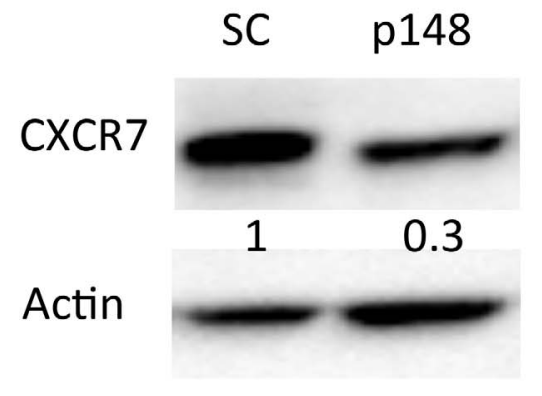

C.

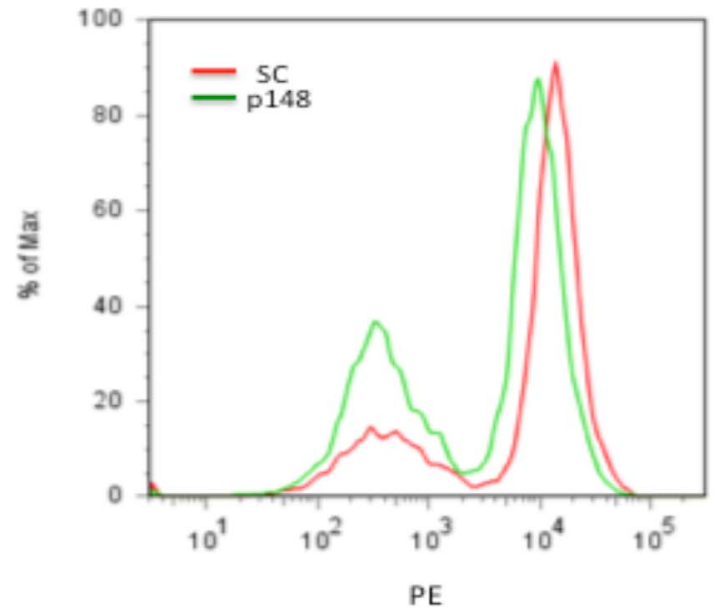

D.

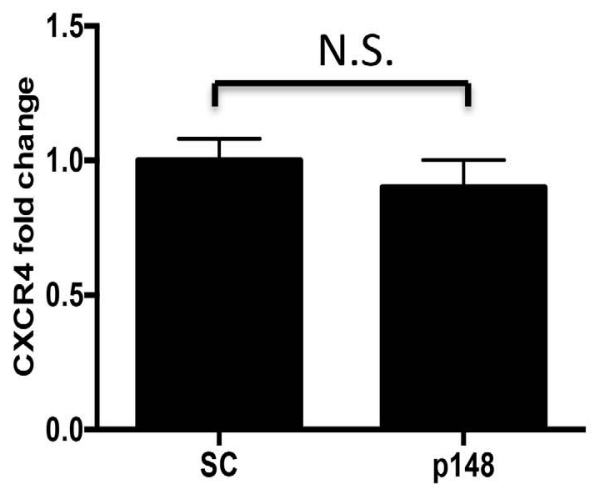

Figure 2. Knockdown of CXCR7 by RNA interference. HBMECs stably infected with CXCR7-targeted shRNA or scrambled control shRNA lentiviruses were subjected to qPCR, Western blot and flow cytometry analysis, respectively, as described in "Materials and Methods". A, Total RNA from shRNA-CXCR7 and shRNA-scramble HBMECs was analyzed for CXCR7 by QPCR. Data are shown as means \pm S.D. from three independent experiments. ${ }^{* *}, p<0.01$. B. Total protein from shRNA-CXCR7 and shRNA-scramble HBMECs was analyzed by Western blot. Three independent experiments were performed and representative data are shown. C. shRNA-scramble and shRNA-CXCR7 HBMECs were collected from 6 well plates and subjected to flow cytometry analysis. Three independent experiments were performed and representative data are shown. $D$. Total RNA from shRNA-CXCR7 and shRNA-scramble HBMECs was analyzed for CXCR4 by qPCR. Data are shown as means \pm S.D from three independent experiments. N.S., not significant. SC: shRNA-scramble HBMECs; p148: shRNA-CXCR7 HBMECs.

doi:10.1371/journal.pone.0103938.g002

Santa Cruz) or mouse anti-GAPDH (1:5000, Abcam) and 1:10,000 secondary antibody.

\section{Flow cytometry analysis}

To examine cell surface expression of CXCR7, shRNACXCR7 or scramble HBMECs were collected and immediately washed with ice-cold Miltenyi Washing Buffer (MWB), then blocked with diluted human FcR blocking reagent (MACS Miltenyi Biotec) for $15 \mathrm{~min}$ at room temperature. Cells were further incubated with human CXCR7-PE or isotype IgG2A-PE for 30 minutes on ice, followed by washing with cold MWB. Samples were read on a FACSAria IIIU (Becton Dickinson, San Jose, CA) and analyzed with FlowJo cytometry analysis software. Ten thousand events were collected per sample.

\section{Cell proliferation assay}

Cell growth was measured using the Cell Proliferation Kit II (Roche Applied Science, Mannheim, Germany) according to manufacturer's protocol. Briefly, shRNA-CXCR7 or scramble HBMECs were seeded at 1000 cells/well in 96-well flat bottom plate (BD Bioscience) in the presence of $200 \mathrm{ng} / \mathrm{ml}$ CXCL12. At the indicated time, $50 \mu \mathrm{l}$ XTT labeling mixture was added and cells were incubated at $37^{\circ} \mathrm{C}$ for 4 hours followed by measuring absorbance at $450 \mathrm{~nm}$ with a reference wavelength at $620 \mathrm{~nm}$ on SpectraMax M2 microplate reader (Molecular Devices).

\section{Tubulogenesis assay}

Tubulogenesis assays were performed as described [27]. Briefly, shRNA-CXCR7 or scramble HMBECs were suspended in 1:10 diluted complete medium (Cell Systems) supplemented with $200 \mathrm{ng} / \mathrm{ml}$ CXCL12. Cells were seeded at $6 \times 10^{4}$ cells/well in 24-well plate coated with $200 \mu \mathrm{l}$ matrigel (BD bioscience, catalogue number: 356237). Six hours later, five high power fields were randomly photographed for each condition and quantified by ImageJ software (NIH, http://rsbweb.nih.gov/ij).

\section{Transwell migration assay}

Transwell migration assays were performed using BD Biocoat growth factor reduced matrigel invasion chambers ( 24 well plate, 8 micron) according to the manufacturer's protocol (BD Bioscience). Briefly, invasion chambers were prehydrated with $500 \mu \mathrm{l} /$ well serum-free medium (Cell Systems) for 2 hours at $37^{\circ} \mathrm{C}$ in $5 \% \mathrm{CO}_{2}$. $2.5 \times 10^{4}$ shRNA-CXCR7 or scramble HBMECs were suspended in $500 \mu \mathrm{l} 10 \%$ complete medium (Cell Systems) and seeded on the 
A.

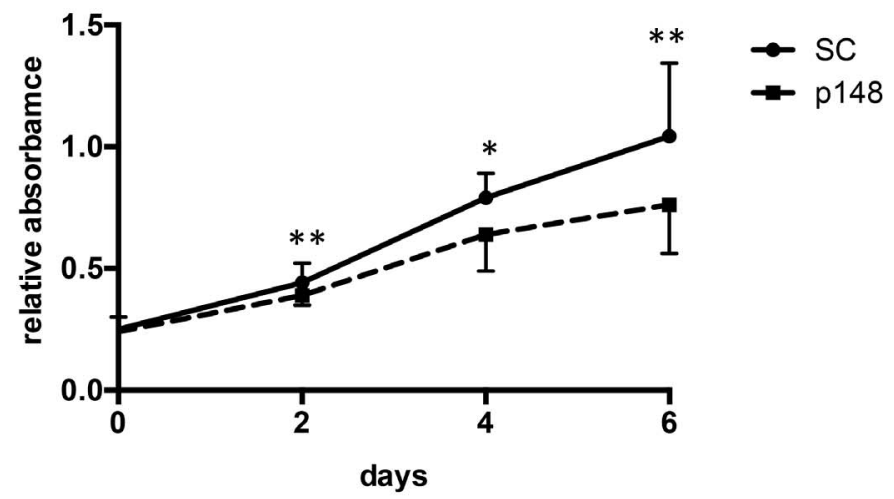

B.
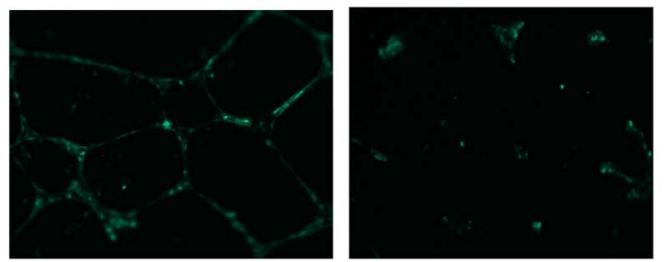

C.
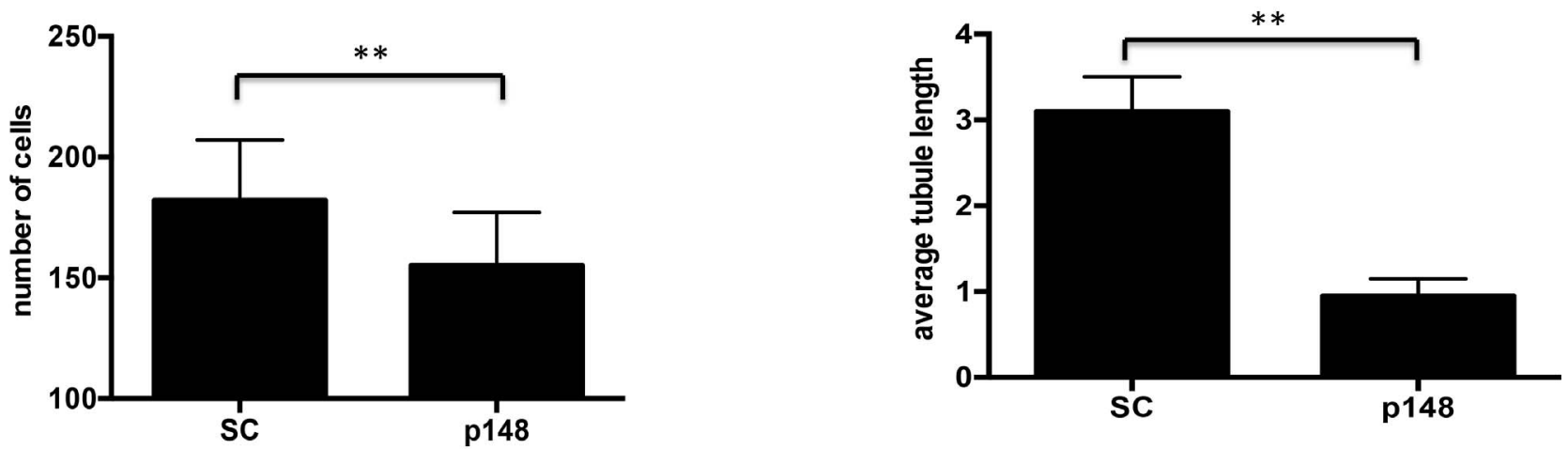

Figure 3. CXCR7 regulates multiple functions in HBMECs. A. shRNA-scramble CXCR7 or shRNA-CXCR7 HBMECs were seeded on 96 well plate in the presence of $200 \mathrm{ng} / \mathrm{ml} \mathrm{CXCL12}$ and cell proliferation was analyzed by XTT assay as described in "Materials and Methods". B. shRNA-scramble or shRNA-CXCR7 HBMECs were seeded on 24 well plate coated with matrigel and tubulogenesis assay was performed as described in in "Materials and Methods". C. shRNA-scramble CXCR7 or shRNA-CXCR7 HBMECs were seeded on the upper chamber of BD matrigel invasion chambers while the lower chamber was filled with $600 \mu \mathrm{l} 10 \%$ complete medium with CXCL12 (200 ng/ml). Cell migration ability was analyzed as described in "Materials and Methods". Data are shown as means \pm S.D from three independent experiments. ${ }^{*}, p<0.05 ;{ }^{* *}, p<0.01 ;$ SC: shRNA-scramble HBMECs; p148: shRNA-CXCR7 HBMECs.

doi:10.1371/journal.pone.0103938.g003

upper chamber while the lower chamber was filled with $600 \mu \mathrm{l}$ $10 \%$ complete medium with CXCL12 (200 ng/ml). Cells were allowed to migrate for 24 hours at $37^{\circ} \mathrm{C}$ in a $5 \% \mathrm{CO}_{2}$ incubator. After 24 hours, cells remaining on the upper membrane surface were removed with a Q-tip and cells remaining on the bottom side of membrane were stained with $10 \mu \mathrm{M}$ cell tracker (Invitrogen). Five high power fields of adherent cells were counted randomly in each well under an inverted microscope and averaged for statistical analysis.

\section{Adhesion assays}

HBMEC adhesion assays were performed as previously described with minor modifications [29]. Briefly, GFP expressing shRNA-CXCR7 or scramble HBMECs were seeded at $8 \times 10^{3} \%$ well in 96 well plates coated with $50 \mu \mathrm{l}$ matrigel (BD Biosciences). After a one hour incubation at $37^{\circ} \mathrm{C}$, adherent cells were washed with PBS, fixed with $3.2 \%$ PFA, and rinsed with PBS again. Adherent cells were quantified by fluorescence on a SpectraMax M2 microplate reader (Molecular Devices). For intercellular adhesion (cell binding) assays, U251MG and U373MG GBM cells were seeded at a density of $1 \times 10^{4} /$ well in a 96 well plate on day 1 . On the second day, tumor cells formed a confluent cell layer and $8 \times 10^{3}$ shRNA-CXCR7 or shRNA scramble HBMECs were seeded on the top of tumor cells and adhesion was measured as described above.

For CXCR7 neutralization and blocking experiments, U251MG and U373MG GBM cells were seeded at a density of
$1 \times 10^{4} /$ well in a 96 well plate on day 1 . On day 2 , HBMECs were stained with carboxyfluorescein succinimidyl ester (CFSE, Molecular Probes) and incubated with $10 \mu \mathrm{g} / \mathrm{ml}$ of CXCR7 specific antibody (11G8), $10 \mu \mathrm{g} / \mathrm{ml}$ control mouse IgG1, $1 \mu \mathrm{M}$ of CXCR7 targeted small molecule antagonist CCX771 (kind gift of Dr. Mark Penfold, Chemocentryx, Inc.) or $1 \mu \mathrm{M}$ control CCX704 (Dr. Penfold) for $30 \mathrm{~min}$. at room temperature. $8 \times 10^{3}$ of HBMECs were then seeded on the top of the tumor cells or matrigel and adhesion was measured as described above.

\section{Statistical analysis}

Results were analyzed by a two-tailed Student's t test and differences with $p<0.05$ were considered to be statistically significant. All experiments were repeated at least three times and data are shown as means \pm S.D.

\section{Results}

\section{CXCR7 is expressed by HBMECs}

In order to study CXCR7 function in brain endothelial cells, we first examined basal expression of CXCR7 in HBMECs by immunocytofluorescence and flow cytometry. As shown in Fig. $1 A$, immunocytofluorescence demonstrated positive cytoplasmic and surface expression of CXCR7 (red) in HBMECs. Flow cytometric analysis of non-permeabilized HBMECs confirmed that GXCR7 was expressed in HBMECs and localized to the cell surface (Fig. 1B). We also performed flow cytometry on permea- 
A. matrigel

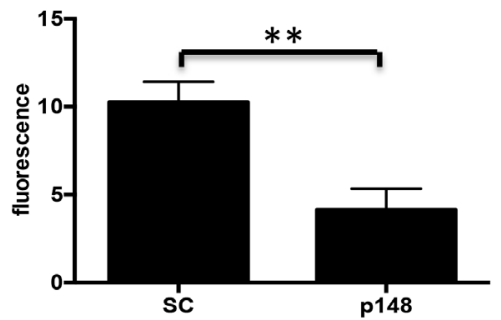

B. matrigel

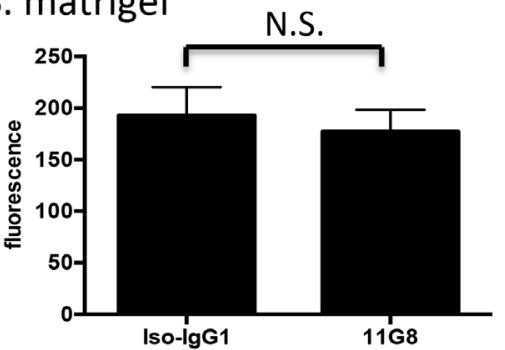

C. matrigel

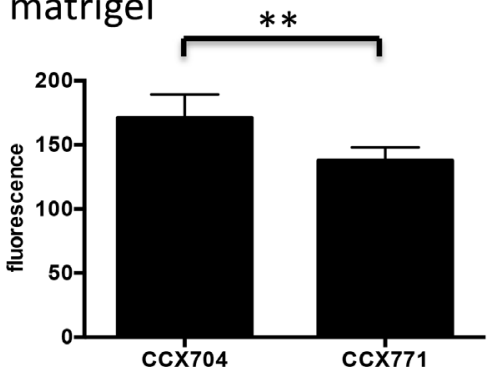

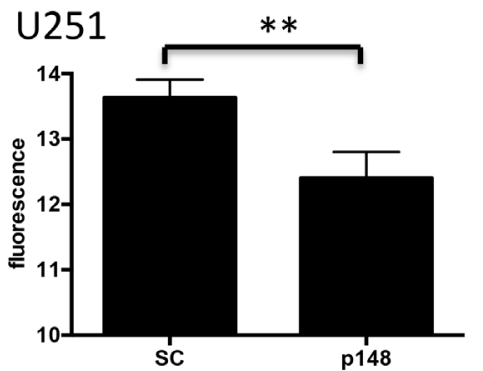

U251

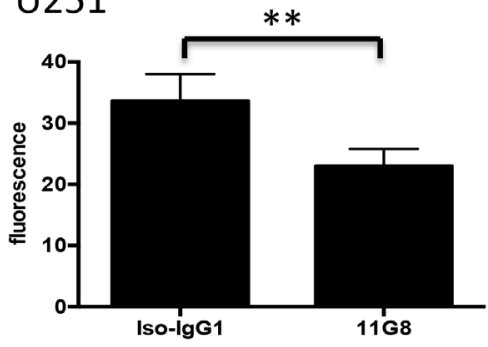

U251

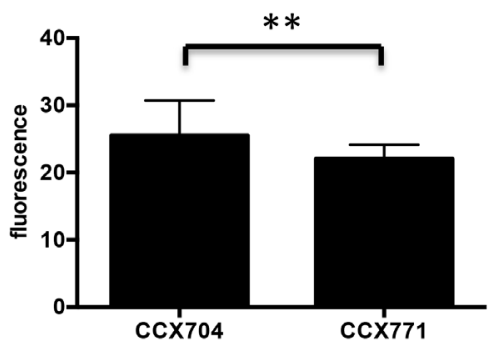

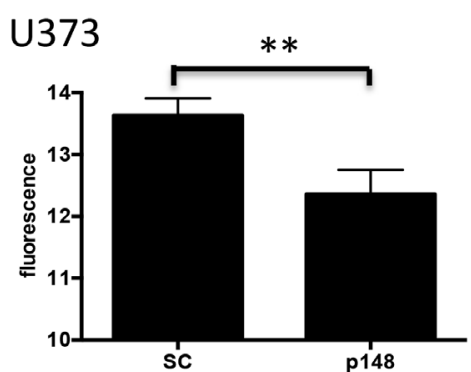

U373

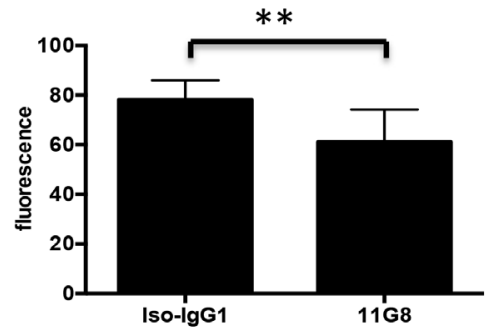

U373

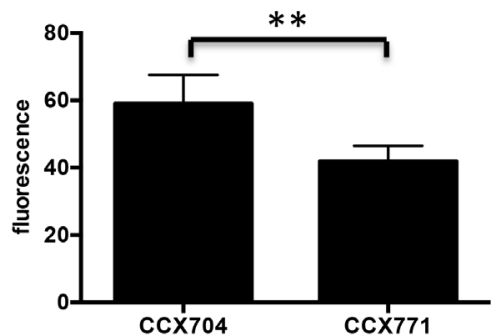

Figure 4. CXCR7 regulates HBMEC adhesion and binding to GBM cells. $A$. shRNA-scramble HBMECs or shRNA-CXCR7 HBMECs were seeded on matrigel or GBM cells (U251 and U373) and adhesion was measured as described in "Materials and Methods". B, HBMECs were pretreated with CXCR7 specific antibody (11G8) or isotype-lgG1 for $30 \mathrm{~min}$. and subsequent adhesion was measured as described in "Materials and Methods". C. HBMECs were pretreated with a CXCR7 small molecule antagonist, CCX771, or negative control, CCX704, for 30 min. and adhesion assays were performed as described in "Materials and Methods". Data are shown as means \pm S.D from three independent experiments. ${ }^{* *}, p<0.01 ; N . S .$, not significant; SC: shRNA-scramble HBMECs; p148: shRNA-CXCR7 HBMECs. doi:10.1371/journal.pone.0103938.g004

bilized cells and found considerable levels of intracellular CXCR7 in HBMECs (data not shown). We did confirm expression of CXCR4 in HBMEGs by qPCR and found that CXCR4 expression was comparable to CXCR7 (data not shown). However, since CXCR4 has been extensively studied [4-7], we focused specifically on the role of CXCR7 in HBMECs.

CXCR7 knockdown attenuates multiple HBMEC functions including proliferation, tube formation, migration, adhesion, and binding to tumor cells

To interrogate the function of CXCR7 in HBMECs, we generated lentiviral constructs expressing shRNA specific to CXCR7 or a nonspecific scrambled control shRNA to suppress CXCR7 in HBMECs. Stably infected HBMECs were selected by puromycin resistance and transgene expression was confirmed by GFP fluorescence. CXCR7 mRNA expression was suppressed over $60 \%$ compared to control, scrambled cells (Fig. $2 A$ ). When the identical lentivirus was used to infect glioma cells, CXCR7 mRNA was suppressed over $90 \%$ (data not shown), indicating that the efficacy of the lentiviral knockdown may depend on cell type.
Suppression of CXCR7 protein was confirmed by Western blot analysis and flow cytometry (Fig. $2 B$ and $2 C$ ). To rule out potential off-target effects of shRNA-CXCR7 on CXCR4, we performed qPCR to check CXCR4 on scramble HBMECs and p148-HBMECs and demonstrated that CXCR4 expression remained unchanged (Fig. 2D).

We next used the stable CXCR7 knockdown and scrambled control HBMEGs (p148-HBMEGs and SC-HBMECs) to study cell proliferation, tubulogenesis, migration, and adhesion. We found that compared to SC-HBMECs, p148-HBMECs showed significant reduction of proliferation even in the presence of CXCL12 (Fig. 3A). The p148-HBMEGs also demonstrated significantly reduced tubulogenesis in the presence of CXCL12, both as measured by tubule number and length (Fig. 3B). Additionally, transwell migration of pl48-HBMECs in response to CXCL12 was significantly reduced (Fig. 3C). Finally, when we used small interfering RNA (siRNA) targeting a non-overlapping sequence of CXCR7 to transiently knockdown CXCR7 in HBMECs, we found that suppression of CXCR7 similarly impeded HBMEC proliferation, tubule formation and migration (data not shown). 
A.

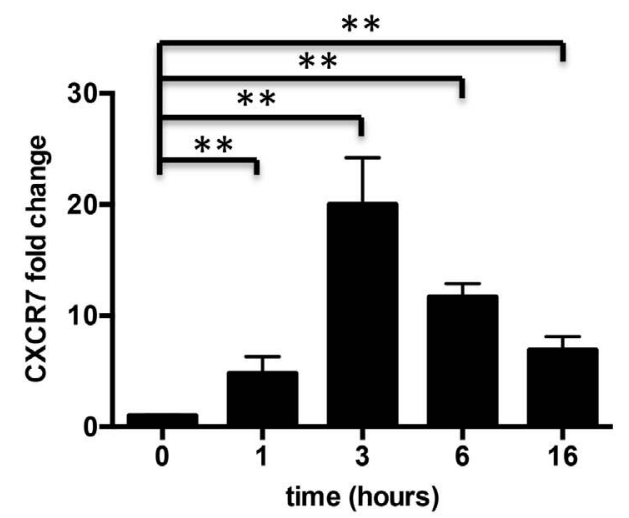

B.

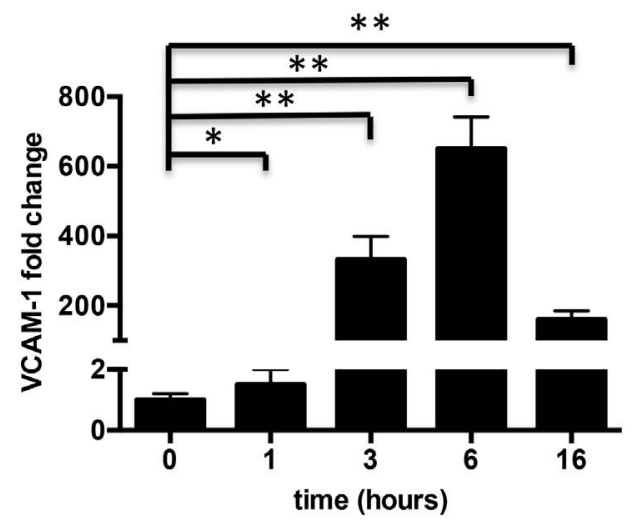

C.

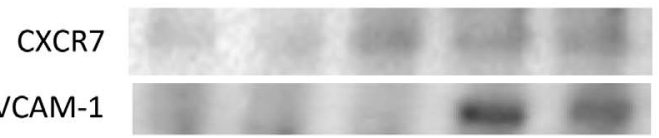

GAPDH

Figure 5. CXCR7 is induced by TNF- $\alpha$ in a time and dose-dependent manner. HBMECs were treated with $10 \mathrm{ng} / \mathrm{ml}$ of TNF- $\alpha$ and collected at $0,1,3,6$, or 16 hours or treated with increasing concentrations $(1,10,100 \mathrm{ng} / \mathrm{ml})$ of TNF- $\alpha$ for 24 hours. $A, B$, and $D$, Total RNA from TNF- $\alpha$-treated HBMECs was analyzed by qPCR as described in "Materials and Methods". Data are shown as means \pm S.D from three independent experiments. *, $p<$ $0.05 ;{ }^{* *}, p<0.01$. $C$ and $E$, Total protein from TNF- $\alpha$-treated HBMECs was analyzed by Western blot as described in "Materials and Methods". Three independent experiments were performed and representative data are shown.

doi:10.1371/journal.pone.0103938.g005

Since GBM is a highly invasive tumor and its cells track along existing blood vessels [30], we examined how HBMECs interacted with extracellular matrix and tumor cells after suppression of CXCR7. We found that suppression of CXCR7 significantly disrupted HBMEG binding to an extracellular matrix as well as GBM cell lines U251 and U373 (Fig. 4A). To confirm these findings, we used a CXCR7 specific antibody, $11 \mathrm{G} 8$, to neutralize surface CXCR7, as well as a small molecule antagonist, CGX771, to block CXCR7 in HBMEGs and measured subsequent cellular adhesion to matrigel and glioma cells. We found that both the blocking antibody and small molecule inhibitor significantly attenuated HBMEC binding to matrigel and tumor cells (Fig. 4B and 4C). Collectively, these data indicate that CXCR7 can actively contribute to adhesion between brain endothelial cells and tumor cells.

CXCR7 can be induced by TNF- $\alpha$ in brain endothelial cells

TNF- $\alpha$ can induce CXCR7 mRNA in HUVEG cells [9,31]. Recognizing that an inflammatory microenvironment contributes
D.

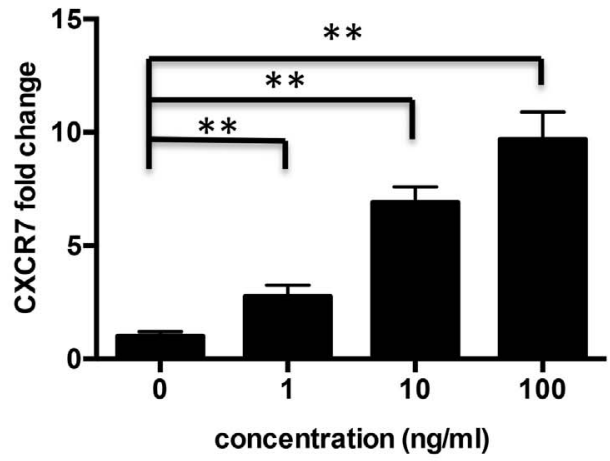

E.

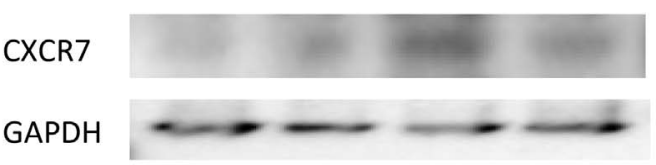

to GBM progression and that TNF- $\alpha$ is a key pro-inflammatory mediator, we asked if TNF- $\alpha$ could regulate CXCR7 in HBMECs. We treated HBMECs with $10 \mathrm{ng} / \mathrm{ml}$ of TNF- $\alpha$ for $0,1,3,6$, or 16 hours or treated with increasing concentrations $(1,10,100 \mathrm{ng} / \mathrm{ml})$ of TNF- $\alpha$ for 24 hours. As shown in Fig. $5 A$, TNF- $\alpha$ treatment $(10 \mathrm{ng} / \mathrm{ml})$ triggered a rapid increase of CXCR7 mRNA in HBMEC cells, beginning as early as 1 hour after treatment and peaking at 3 hours after addition. This was confirmed by Western blot (Fig. 5C). Lower doses of TNF- $\alpha(1 \mathrm{ng} / \mathrm{ml})$ induced CXCR7 to a lesser degree at both the mRNA and protein levels (Fig. $5 D$ and $5 E$ ). Flow cytometry verified that TNF- $\alpha$ induced CXCR7 protein (data not shown). Overall, GXCR7 was up-regulated by $\mathrm{TNF}-\alpha$ in a time and dose-dependent manner.

\section{CXCR7 is an upstream gene of VCAM-1}

VCAM-1 is a key mediator of intercellular contact in tumors and has been suggested as a potential therapeutic target in cancer metastasis [32] and GBM cell invasion [33]. Since CXCR7 knockdown significantly suppressed HBMEC adhesion to matrigel 
A.
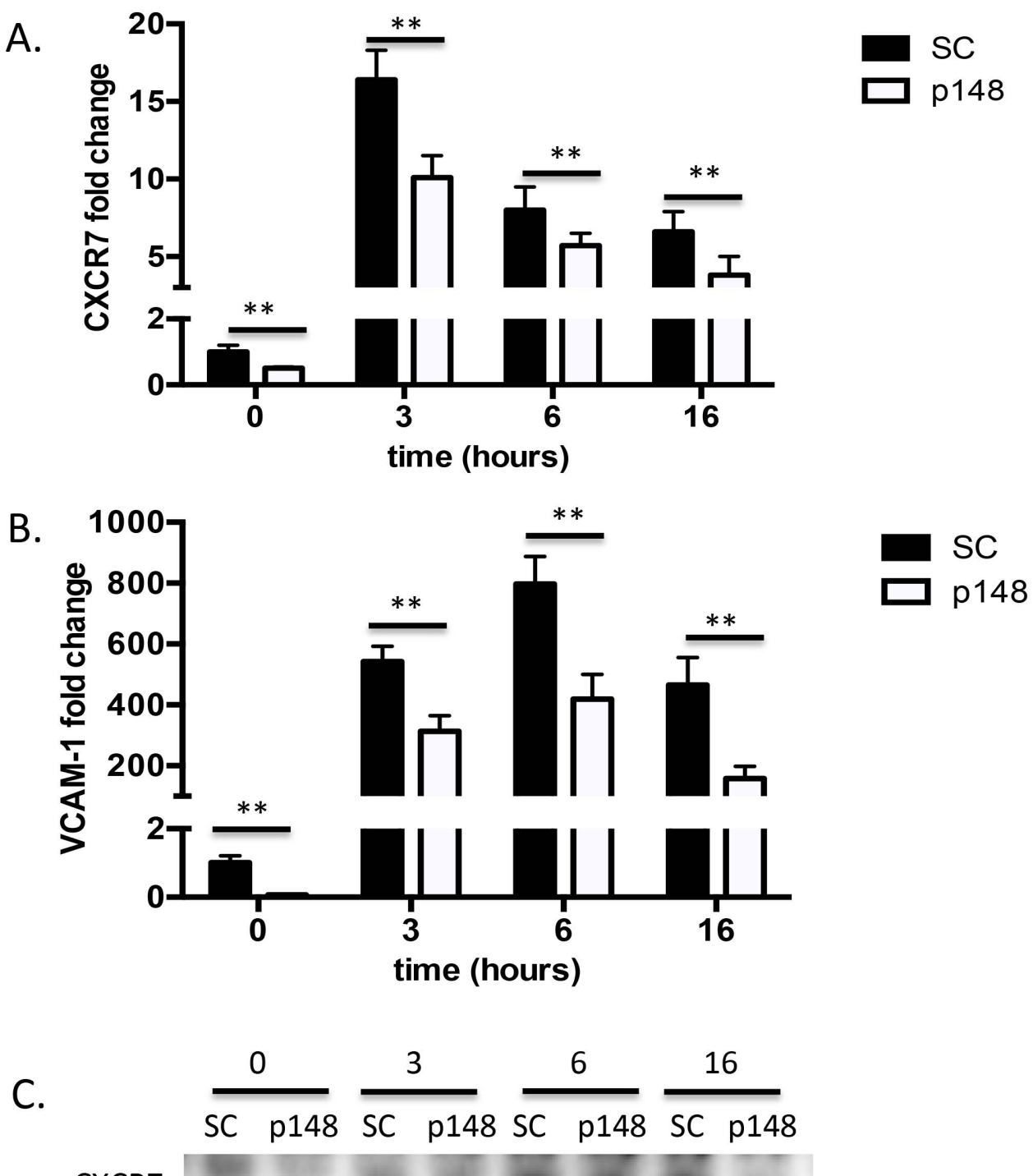

CXCR7

VCAM-1

GAPDH

Figure 6. CXCR7 is an upstream gene of VCAM-1 in HBMECs. shRNA-scramble or shRNA-CXCR7 scramble HBMECs were treated with $10 \mathrm{ng} / \mathrm{ml}$ TNF- $\alpha$ and collected at indicated time points. $A$ and $B$. Total RNA from TNF- $\alpha$-treated shRNA-scramble or shRNA-CXCR7 HBMECs was analyzed by qPCR as described in "Materials and Methods". Data are shown as means \pm S.D from three independent experiments. **, $p<0.01$. C, Total protein from TNF- $\alpha$-treated shRNA-CXCR7 or scramble HBMECs was analyzed by Western blot as described in "Materials and Methods". Three independent experiments were performed and representative data are shown. SC: shRNA-scramble HBMECs; p148: shRNA-CXCR7 HBMECs.

doi:10.1371/journal.pone.0103938.g006

and glioma cell lines, we questioned whether this could be due to a coordinate change in VCAM-1 levels. In response to TNF- $\alpha$ treatment, CXCR7 expression increased, peaking at 3 hours, followed by a peak induction of VCAM-1 expression at 6 hours (Fig. 5A-C). Subsequently, we asked if CXCR7 could regulate VCAM-1 in HBMEGs. We found that suppression of CXCR7 by shRNA blocked the induction of VCAM-1 by TNF- $\alpha$ at both mRNA and protein levels (Fig. 6). Collectively, these data suggest that CXCR7 functions upstream of VCAM-1 in HBMECs.

\section{Discussion}

Malignant gliomas are characterized by abundant neovascularization, a hallmark that correlates directly with clinical recurrence and inversely with the post-operative survival. Radiation and chemotherapy can damage tumor vasculature and inhibit tumor angiogenesis. Recovery of the tumor microvasculature is mediated, in part, by CXCL12 and CXCR4 [34,35], but the role of receptor CXCR7 remains controversial. We and other groups found that CXCR7 was highly expressed in the malignant brain tumor vasculature [18-20,23], increased with brain tumor grade [20,21] 
and correlated with poor survival in glioma patients (Liu Y, unpublished data). However, despite the fact that CXCR7 is highly expressed in tumor vasculature, the functional role of CXCR7 in brain endothelial cells remains poorly understood. Although two recent studies showed that CXCR7 expression is sufficient to drive pulmonary and blood vascular endothelial cell growth [14,36], these results are not necessarily translatable to the cerebrovasculature, which is characterized by unique blood brain barrier biology and gene expression profiles. In this report, we used HBMECs to study the role of CXCR7 in normal brain endothelial cells in vitro. We confirmed that CXCR7 was expressed on HBMEGs (Fig. 1), consistent with a recent report [37]. Furthermore, we found that suppression of CXCR7 by targeted shRNA significantly inhibited multiple aspects of brain endothelial cell function including proliferation, tube formation, migration and adhesion (Fig. 3-4). To validate these findings, we demonstrated that transient knockdown of CXCR7 by siRNA in HBMECs led to similarly significant reductions in proliferation, tube formation, and migration (data not shown). Since tubulogenesis, unlike proliferation, is measured in hours, the decrease seen in tubule formation was due to altered acute cellular responses to the microenvironment, not long term growth retardation. Furthermore, inhibition of HBMEC responses to CXCL12 was observed despite the continued expression of CXCR4 in these cells. Collectively, these data suggest that CXCR7 actively participates in brain microvascular endothelial cell growth and angiogenesis. We also found that CXCR4 expression level in HBMECs is comparable to CXCR7 and blockage of CXCR4 with its antagonist AMD3100 significantly impaired HBMEGs tube formation and transwell migration (data not shown). Thus, selectively targeting both CXCR7 and CXCR4 in tumor vasculature may provide a robust anti-angiogenic approach to GBM treatment.

The CXCL12 pathway is a key receptor system in the cross-talk between tumor cells and the vasculature in glioma [37-39]. Increased CXCL12/CXCR4 signaling has been reported after withdrawal of chemotherapy and shown to promote bone marrow derived endothelial progenitor cell mobilization and subsequent tumor homing, leading to an angiogenic rebound and regrowth of tumors $[34,40,41]$. CXCR7 has been suggested to regulate cell adhesion in tumor cells $[9,42,43]$, endothelial progenitor cells $[44,45]$ and renal multipotent progenitors [46]. In the central nervous system, CXCR7 was shown to modulate the adhesion of leukocytes to the microvasculature [15]. Recently, it was proposed that inhibition of CXCR7 on endothelial cells coupled with inhibition of CXCR4 on CD1 lb+ monocytes can inhibit the postirradiation recovery of the vasculature and delay local tumor recurrence [35]. In this study we showed that stable knockdown of

\section{References}

1. Mirshahi F, Pourtau J, Li H, Muraine M, Trochon V, et al. (2000) SDF-1 activity on microvascular endothelial cells: consequences on angiogenesis in in vitro and in vivo models. Thromb Res 99: 587-594.

2. Molino M, Woolkalis MJ, Prevost N, Pratico D, Barnathan ES, et al. (2000) CXCR4 on human endothelial cells can serve as both a mediator of biological responses and as a receptor for HIV-2. Biochim Biophys Acta 1500: 227-240.

3. Kanda S, Mochizuki Y, Kanetake H (2003) Stromal cell-derived factor-1alpha induces tube-like structure formation of endothelial cells through phosphoinositide 3-kinase. J Biol Chem 278: 257-262.

4. Zou YR, Kottmann AH, Kuroda M, Taniuchi I, Littman DR (1998) Function of the chemokine receptor CXCR4 in haematopoiesis and in cerebellar development. Nature 393: 595-599.

5. Tachibana K, Hirota S, Iizasa H, Yoshida H, Kawabata K, et al. (1998) The chemokine receptor CXCR4 is essential for vascularization of the gastrointestinal tract. Nature 393: 591-594.

6. Salcedo R, Wasserman K, Young HA, Grimm MC, Howard OM, et al. (1999) Vascular endothelial growth factor and basic fibroblast growth factor induce
CXCR7 on HBMECs significantly inhibited brain endothelial cell binding to tumor cells (Fig. 4A). Likewise, blocking CXCR7 with a neutralizing antibody (11G8) or small molecule antagonist (CCX771) similarly reduced HBMEC binding ability to matrigel and GBM cells (Fig. $4 B$ and $4 C$ ). This suggests that CXCR7 actively contributes to physical interactions between brain endothelial cells and their environment and may contribute to glioma cell invasion along vascular tracks. Disruption of endothelial-tumor cell adhesion may be a mechanism to prevent GBM invasion as well as recurrence.

TNF- $\alpha$ can directly and indirectly promote GBM tumorigenesis and angiogenesis $[47,48]$. TNF- $\alpha$ expression levels can be elevated in GBM endothelial cells [49]. We examined whether TNF- $\alpha$ induced CXCR7 in HBMECs. We found that TNF- $\alpha$ induced CXCR7 expression in HBMECs at both the mRNA and protein level in a time- and dose-dependent manner (Fig. 5), indicating crosstalk between the TNF- $\alpha$ and CXCL12 signaling pathways.

Our results also reveal that CXCR7 can regulate VCAM-1 in HBMECs. CXCR7 has been reported to influence adhesion in multiple cell types and can specifically regulate cadherin 11 and CD44 in prostate cancer cells [42]. We found that suppression of CXCR7 significantly repressed VCAM-1 expression at both mRNA and protein levels with and without TNF- $\alpha$ stimulation (Fig. 6). Thus, CXCR7 may regulate endothelial adhesion, at least in part, through modulation of downstream VCAM-1 levels and targeting CXCR7/VCAM-1 may provide a novel opportunity to prevent tumor invasion and metastasis.

In conclusion, our study demonstrates that CXCR7 can regulate multiple proliferative functions in brain endothelial cells and can, itself, be regulated by TNF- $\alpha$. CXCR7 mediates endothelial cell adhesion to GBM cells and endothelial expression of the adhesion molecule, VCAM-1. Targeted inhibition of vascular CXCR7 may have direct anti-angiogenic or antimetastatic benefits for brain tumor patients.

\section{Acknowledgments}

We thank Dr. Naxin Guo of University of Rochester for technical assistance and advice. We thank Dr. Abdellatif Benraiss for assistance with lentiviruses and Dr. Su Wang and Devin Chandler-Militello for help with flow cytometry. We also thank Dr. Mark Penfold (Chemocentryx, Inc) for providing CXCR7 antagonist CCX771 and control CCX704.

\section{Author Contributions}

Conceived and designed the experiments: YL ECW KAW. Performed the experiments: YL. Analyzed the data: YL. Contributed reagents/materials/ analysis tools: YL ECW KAW. Contributed to the writing of the manuscript: YL ECW KAW.

expression of CXCR4 on human endothelial cells: In vivo neovascularization induced by stromal-derived factor-1alpha. Am J Pathol 154: 1125-1135.

7. Salcedo R, Oppenheim JJ (2003) Role of chemokines in angiogenesis: CXCL12/ SDF-1 and CXCR4 interaction, a key regulator of endothelial cell responses. Microcirculation 10: 359-370.

8. Balabanian K, Lagane B, Infantino S, Chow KY, Harriague J, et al. (2005) The chemokine SDF-1/CXCL12 binds to and signals through the orphan receptor RDC1 in T lymphocytes. J Biol Chem 280: 35760-35766.

9. Burns JM, Summers BC, Wang Y, Melikian A, Berahovich R, et al. (2006) A novel chemokine receptor for SDF-1 and I-TAC involved in cell survival, cell adhesion, and tumor development. J Exp Med 203: 2201-2213.

10. Naumann U, Cameroni E, Pruenster M, Mahabaleshwar H, Raz E, et al. (2010) CXCR7 functions as a scavenger for CXCL12 and CXCL11. PLoS One 5: e9175.

11. Hattermann K, Held-Feindt J, Lucius R, Muerkoster SS, Penfold ME, et al. (2010) The chemokine receptor CXCR7 is highly expressed in human glioma cells and mediates antiapoptotic effects. Cancer Res 70: 3299-3308. 
12. Watanabe K, Penfold ME, Matsuda A, Ohyanagi N, Kaneko K, et al. (2010) Pathogenic role of CXCR7 in rheumatoid arthritis. Arthritis Rheum 62: 3211 3220 .

13. Schutyser E, Su Y, Yu Y, Gouwy M, Zaja-Milatovic S, et al. (2007) Hypoxia enhances CXCR4 expression in human microvascular endothelial cells and human melanoma cells. Eur Cytokine Netw 18: 59-70.

14. Costello CM, McCullagh B, Howell K, Sands M, Belperio JA, et al. (2012) A role for the CXCL12 receptor, CXCR7, in the pathogenesis of human pulmonary vascular disease. Eur Respir J 39: 1415-1424.

15. Cruz-Orengo L, Holman DW, Dorsey D, Zhou L, Zhang P, et al. (2011) CXCR7 influences leukocyte entry into the CNS parenchyma by controlling abluminal CXCL12 abundance during autoimmunity. J Exp Med 208: 327339.

16. Sierro F, Biben C, Martinez-Munoz L, Mellado M, Ransohoff RM, et al. (2007) Disrupted cardiac development but normal hematopoiesis in mice deficient in the second CXCL12/SDF-1 receptor, CXCR7. Proc Natl Acad Sci U S A 104: 14759-14764.

17. Gerrits H, van Ingen Schenau DS, Bakker NE, van Disseldorp AJ, Strik A, et al. (2008) Early postnatal lethality and cardiovascular defects in CXCR7-deficient mice. Genesis 46: 235-245.

18. Madden SL, Cook BP, Nacht M, Weber WD, Callahan MR, et al. (2004) Vascular gene expression in nonneoplastic and malignant brain. Am J Pathol 165: 601-608.

19. Liu Y, Carson-Walter EB, Cooper A, Winans BN, Johnson MD, et al. (2010) Vascular gene expression patterns are conserved in primary and metastatic brain tumors. J Neurooncol 99: 13-24.

20. Wurth R, Barbieri F, Bajetto A, Pattarozzi A, Gatti M, et al. (2011) Expression of CXCR7 chemokine receptor in human meningioma cells and in intratumoral microvasculature. J Neuroimmunol 234: 115-123.

21. Walters MJ, Ebsworth K, Berahovich RD, Penfold ME, Liu SC, et al. (2014) Inhibition of CXCR7 extends survival following irradiation of brain tumours in mice and rats. $\mathrm{Br} \mathrm{J}$ Cancer.

22. Miao Z, Luker KE, Summers BC, Berahovich R, Bhojani MS, et al. (2007) CXCR7 (RDC1) promotes breast and lung tumor growth in vivo and is expressed on tumor-associated vasculature. Proc Natl Acad Sci U S A 104: 15735-15740.

23. Takano S, Yamashita T, Ohneda O (2010) Molecular therapeutic targets for glioma angiogenesis. J Oncol 2010: 351908.

24. Monnier J, Boissan M, L'Helgoualc'h A, Lacombe ML, Turlin B, et al. (2012) CXCR7 is up-regulated in human and murine hepatocellular carcinoma and is specifically expressed by endothelial cells. Eur J Cancer 48: 138-148.

25. Luker KE, Lewin SA, Mihalko LA, Schmidt BT, Winkler JS, et al. (2012) Scavenging of CXCL12 by CXCR7 promotes tumor growth and metastasis of CXCR4-positive breast cancer cells. Oncogene 31: 4750-4758.

26. Salmaggi A, Maderna E, Calatozzolo C, Gaviani P, Canazza A, et al. (2009) CXCL12, CXCR4 and CXCR7 expression in brain metastases. Cancer Biol Ther 8: 1608-1614.

27. Carson-Walter EB, Hampton J, Shue E, Geynisman DM, Pillai PK, et al. (2005) Plasmalemmal vesicle associated protein-1 is a novel marker implicated in brain tumor angiogenesis. Clin Cancer Res 11: 7643-7650.

28. Glushakova O, Kosugi T, Roncal C, Mu W, Heinig M, et al. (2008) Fructose induces the inflammatory molecule ICAM-1 in endothelial cells. J Am Soc Nephrol 19: 1712-1720.

29. Jung H, Lee KP, Park SJ, Park JH, Jang YS, et al. (2008) TMPRSS4 promotes invasion, migration and metastasis of human tumor cells by facilitating an epithelial-mesenchymal transition. Oncogene 27: 2635-2647.

30. Bergers G, Benjamin LE (2003) Tumorigenesis and the angiogenic switch. Nat Rev Cancer 3: 401-410.
31. Zhou J, Jin Y, Gao Y, Wang H, Hu G, et al. (2002) Genomic-scale analysis of gene expression profiles in TNF-alpha treated human umbilical vein endothelial cells. Inflamm Res 51: 332-341.

32. Chen Q Massague J (2012) Molecular pathways: VCAM-1 as a potential therapeutic target in metastasis. Clin Cancer Res 18: 5520-5525.

33. Zheng Y, Yang W, Aldape K, He J, Lu Z (2013) Epidermal growth factor (EGF)enhanced vascular cell adhesion molecule-1 (VCAM-1) expression promotes macrophage and glioblastoma cell interaction and tumor cell invasion. J Biol Chem 288: 31488-31495.

34. Kioi M, Vogel H, Schultz G, Hoffman RM, Harsh GR, et al. (2010) Inhibition of vasculogenesis, but not angiogenesis, prevents the recurrence of glioblastoma after irradiation in mice. J Clin Invest 120: 694-705.

35. Martin BJ (2013) Inhibiting vasculogenesis after radiation: a new paradigm to improve local control by radiotherapy. Semin Radiat Oncol 23: 281-287.

36. Totonchy JE, Osborn JM, Botto S, Clepper L, Moses AV (2013) Aberrant proliferation in CXCR7+ endothelial cells via degradation of the retinoblastoma protein. PLoS One 8: e69828.

37. Rao S, Sengupta R, Choe EJ, Woerner BM, Jackson E, et al. (2012) CXCL12 mediates trophic interactions between endothelial and tumor cells in glioblastoma. PLoS One 7: e33005.

38. Zagzag D, Esencay M, Mendez O, Yee H, Smirnova I, et al. (2008) Hypoxiaand vascular endothelial growth factor-induced stromal cell-derived factorlalpha/CXCR4 expression in glioblastomas: one plausible explanation of Scherer's structures. Am J Pathol 173: 545-560.

39. Kenig S, Alonso MB, Mueller MM, Lah TT (2010) Glioblastoma and endothelial cells cross-talk, mediated by SDF-1, enhances tumour invasion and endothelial proliferation by increasing expression of cathepsins $\mathrm{B}, \mathrm{S}$, and MMP-9. Cancer Lett 289: 53-61.

40. Shaked Y, Henke E, Roodhart JM, Mancuso P, Langenberg MH, et al. (2008) Rapid chemotherapy-induced acute endothelial progenitor cell mobilization: implications for antiangiogenic drugs as chemosensitizing agents. Cancer Cell 14: 263-273.

41. Aghi M, Cohen KS, Klein RJ, Scadden DT, Chiocca EA (2006) Tumor stromalderived factor-1 recruits vascular progenitors to mitotic neovasculature, where microenvironment influences their differentiated phenotypes. Cancer Res 66: 9054-9064.

42. Wang J, Shiozawa Y, Wang J, Wang Y, Jung Y, et al. (2008) The role of CXCR7/RDC1 as a chemokine receptor for CXCL12/SDF-1 in prostate cancer. J Biol Chem 283: 4283-4294.

43. Zheng K, Li HY, Su XL, Wang XY, Tian T, et al. (2010) Chemokine receptor CXCR7 regulates the invasion, angiogenesis and tumor growth of human hepatocellular carcinoma cells. J Exp Clin Cancer Res 29: 31.

44. Dai X, Tan Y, Cai S, Xiong X, Wang L, et al. (2011) The role of CXCR7 on the adhesion, proliferation and angiogenesis of endothelial progenitor cells. J Cell Mol Med 15: 1299-1309.

45. Yan X, Cai S, Xiong X, Sun W, Dai X, et al. (2012) Chemokine receptor CXCR7 mediates human endothelial progenitor cells survival, angiogenesis, but not proliferation. J Cell Biochem 113: 1437-1446.

46. Mazzinghi B, Ronconi E, Lazzeri E, Sagrinati C, Ballerini L, et al. (2008) Essential but differential role for CXCR4 and CXCR7 in the therapeutic homing of human renal progenitor cells. J Exp Med 205: 479-490.

47. Radeff-Huang J, Seasholtz TM, Chang JW, Smith JM, Walsh CT, et al. (2007) Tumor necrosis factor-alpha-stimulated cell proliferation is mediated through sphingosine kinase-dependent Akt activation and cyclin D expression. J Biol Chem 282: 863-870.

48. Brat DJ, Bellail AC, Van Meir EG (2005) The role of interleukin-8 and its receptors in gliomagenesis and tumoral angiogenesis. Neuro Oncol 7: 122-133.

49. Huang P, Rani MR, Ahluwalia MS, Bae E, Prayson RA, et al. (2012) Endothelial expression of TNF receptor-1 generates a proapoptotic signal inhibited by integrin alpha6betal in glioblastoma. Cancer Res 72: 1428-1437. 